\title{
THE CANONICAL FORM OF A SCALAR OPERATOR ON A BANACH SPACE
}

\author{
G. D. FAULKNER AND J. E. HUNEYCUTT, JR.
}

\begin{abstract}
Let $A=\int \lambda d E(\lambda)$ be a scalar operator on a Banach space $X$. If there exists a vector $g \in X$ such that the closed convex hull of the range of the vector measure $\mu(\cdot)=E(\cdot) g$ has nonvoid interior, then $A$ is similar to the operator $Q f(\lambda)=\lambda f(\lambda)$ on a quotient space of a suitably constructed $\mathfrak{E}^{\infty}$ space.
\end{abstract}

I. Introduction. Let $A$ be a bounded, selfadjoint operator on the Hilbert space $H$ with spectral representation $A=\int \lambda d E(\lambda)$. If $A$ has simple spectra (i.e. there is some $g \in H$ so that the linear span of the range of the vector measure $\mu(\cdot)=E(\cdot) g$ is dense in $H$ ), then $A$ is unitarily equivalent to multiplication by the independent variable on $\mathcal{L}^{2}(\sigma(A), \nu)$, where $\nu(M)=$ $\langle\mu(M), g\rangle[1$, p. 52]. The condition that $A$ have simple spectra is in some sense a requirement that the range of the vector measure $E(\cdot) g$ be well dispersed in $H$. In order to motivate an alternative condition on the dispersion of the range of a vector measure we consider the following example. Let $Q$ be the operation of multiplication by the independent variable on $\mathfrak{L}^{\infty}([0,1], \mu)$, i.e. $(Q f)(\lambda)=\lambda f(\lambda)$. The operator $Q$ is a prespectral operator with spectral family $E(M) g=1_{M} \cdot g$. Let $\mu(M)=E(M) 1$, then the range of $\mu, \mathcal{R}(\mu)$, is the set of characteristic functions of measurable subsets of $[0,1]$. The positive portion of the unit ball of $\mathfrak{L}^{\infty}([0,1], \mu)$ is weak-* compact and the range of $\mu$ coincides with its extreme points. Therefore, the closed, convex hull of $\mathcal{R}(\mu)$ is all of the positive part of the ball; and thus, has nonvoid interior. We will say that a vector measure $\mu$, taking values in a Banach space, is full provided $\{\overline{\operatorname{co}}(\Re(\mu))\}^{0} \neq \varnothing$.

In this paper we shall show that, for a scalar operator $A=\int \lambda d E(\lambda)$ defined on a Banach space $X$, the existence of a vector $g \in X$ such that $\mu(\cdot)=E(\cdot) g$ is full implies that $A$ is similar to $Q f(\lambda)=\lambda f(\lambda)$ on a quotient space of a suitably constructed $\mathfrak{L}^{\infty}$ space.

II.

Definition 1. Let $\mu$ be a vector valued measure defined on a $\sigma$-algebra $\mathscr{B}$ and taking values in a Banach space $X$ with norm $|\cdot|$. The semivariation of $\mu$, denoted by $\|\mu\|$, is the scalar valued function on $\mathscr{B}$ defined by

Received by the editors February 8, 1977 and, in revised form, October 31, 1977. AMS (MOS) subject classifications (1970). Primary 47A65, 47B40, 46G10, $28 \mathrm{~A} 45$.

Key words and phrases. Scalar operator, vector measures. 


$$
\|\mu\|(E)=\sup \left|\sum_{i=0}^{n} \alpha_{i} \mu\left(E_{i}\right)\right|
$$

where the supremum is taken over all finite collections of scalars with $\left|\alpha_{i}\right| \leqslant 1$ and all finite partitions of $E$ into disjoint members of $\mathscr{B}$.

The support of $\mu$ will be denoted by $\operatorname{supp}(\mu)$.

LEMMA $1[2$, p. 320]. Let $\mu$ be a vector valued measure defined on a $\sigma$-algebra B. Then

(1) $\|\mu\|(E) \geqslant|\mu(E)| \geqslant 0$,

(2) $\|\mu\|(E) \leqslant 4 \sup _{F \subseteq E}|\mu(F)|<\infty$,

(3) $\|\mu\|(E) \leqslant\|\mu\|(F)$ if $E \subseteq F$,

(4) there is a positive scalar measure $\lambda$ defined on $\Re$ so that

(a) $\lambda(E) \leqslant\|\mu\|(E)$

(b) $\lim _{\lambda(E) \rightarrow 0}\|\mu\|(E)=0$.

In all that follows $\lambda$ will be a scalar measure guaranteed by (4) above. By (4) (b) of the lemma, $\lambda(E)=0$ if and only if $\|\mu\|(E)=0$, so for $F \in \mathscr{B}$, $\mu-\operatorname{ess} \sup _{s \in F}|f(s)|=\lambda$ - ess $\sup _{s \in F}|f(s)|$; thus we denote by $\mathcal{L}^{\infty}(\mu)$, the space $\mathscr{L}^{\infty}(\lambda)$. The reason for this apparently artificial device is the desire to associate the space of functions with the measure $\mu$ itself.

Definition 2. Let $\mu$ be a vector valued measure defined on a $\sigma$-algebra $\mathscr{B}$ of subsets of a set $T$. We denote by $\mathcal{L}^{1}(T, \mu)$ the set of all measurable complex valued functions $f$ defined on $T$ for which $\int f d \mu$ exists. For $f \in \mathcal{L}^{1}(T, \mu)$, $\mu_{f}(E)=\int_{E} f d \mu$ defines a vector measure on $\mathscr{B}$. The norm on $\mathcal{L}^{1}(T, \mu)$ is then defined by

$$
\|f\|_{1}=\left\|\mu_{f}\right\|(T) \text {. }
$$

In the case that $\mu$ is scalar valued this space of functions is equivalent to the usual $L^{1}$ space.

By $[3$, p. 45$] \mathcal{L}^{\prime}(T, \mu)$ is complete. In addition since $\lambda(E) \leqslant\|\mu\|(E)<\infty$ and

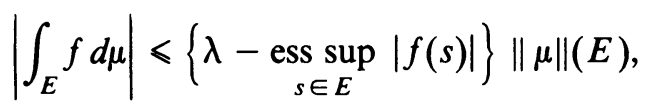

$\mathfrak{L}^{\infty}(\mu) \subset \mathcal{L}^{1}(T, \mu)$.

Let $C(T)$ denote the continuous complex valued functions on a subset $T$ of the complex plane. We then have

THEOREM 1. $C(T)$ is dense in $\mathrm{L}^{\mathrm{l}}(T, \mu)$.

Proof. Let $A \in \Re$. By Lusin's theorem there exists a sequence of continuous functions $g_{n}$, with $\left|g_{n}(s)\right| \leqslant 1$, such that for $A_{n}=\left\{s \in T \mid g_{n}(s) \neq\right.$ $\left.1_{A}(s)\right\}, \lim _{n \rightarrow \infty} \lambda\left(A_{n}\right)=0$. It follows that for $\epsilon>0$ there exists an integer $N$ so that for $n \geqslant N,\|\mu\|\left(A_{n}\right) \leqslant \epsilon / 8$. For this $N$ and $n \geqslant N$ we have 


$$
\begin{aligned}
\left\|1_{A}-g_{n}\right\|_{1} & \leqslant 4 \sup _{F \subseteq T}\left|\int_{F}\left(1_{A}-g_{n}\right) d \mu\right|=4 \sup _{F \subseteq T}\left|\int_{F \cap A_{n}}\left(1-g_{n}\right) d \mu\right| \\
& \leqslant 8 \sup _{F \subseteq T}\|\mu\|\left(F \cap A_{n}\right) \leqslant \epsilon .
\end{aligned}
$$

Thus, since simple functions are dense in $\mathfrak{L}^{1}(T, \mu)$, the theorem holds.

We note that since

$$
\|f\|_{1} \leqslant 4 \sup _{F \subseteq T}\left|\int_{F} f d \mu\right| \leqslant 4\{\lambda-\underset{s \in T}{\operatorname{ess} \sup |f(s)|\}\|\mu\|(T),}
$$

it follows that the polynomials together with their conjugates are dense in $\mathcal{L}^{\prime}(T, \mu)$ whenever $T$ is a compact subset of the complex plane.

LEMMA 2 [3, p. 76]. If $\mu$ is a vector valued measure defined on a o-algebra $\mathscr{B}$, taking values in a Banach space $X$, then

$$
\overline{\text { co }} \mathscr{R}(\mu)=\left\{\int f d \mu \mid f \text { is measurable and } f(s) \in[0,1] \text { for all } s \in T\right\} \text {. }
$$

LEMMA 3. Let $\mu$ be a vector measure defined on a $\sigma$-algebra $\mathscr{B}$ of subsets of a set $T$, taking values in a Banach space $X$ with norm $|\cdot|$. Then for every complex valued function defined on $T$, with $|f(s)| \leqslant 1$ for all $s \in T$, we have

$$
\left|\int_{E} f(s) d \mu(s)\right| \leqslant\|\mu\|(E) .
$$

The proof is standard, using measurable disjoint partitions of $E$.

THEOREM 2. The linear operator $Q: \mathcal{L}^{1}(T, \mu) \rightarrow \mathcal{L}^{1}(T, \mu)$ defined by $Q f(s)=$ $s f(s)$ is continuous if $\operatorname{supp}(\mu)$ is contained in a bounded subset of the complex plane.

Proof. Clearly for $f \in \mathcal{L}^{1}, Q f \in \mathcal{L}^{1}$ so that the operator has domain all of $\mathcal{L}^{1}$. Now suppose supp $(\mu) \subseteq\{z \in \mathbf{C}|| z \mid \leqslant r\}=D(r)$. Then for $E \subseteq D(r)$,

$$
\left|\int_{E} s f(s) d \mu(s)\right|=\left|\int_{E} s d \mu_{f}(s)\right|=r\left|\int_{E} \frac{s}{r} d \mu_{f}(s)\right| .
$$

Now $f(s)=s / r$ satisfies $|f(s)| \leqslant 1$, so by Lemma 3

$$
\left|\int_{E} s f(s) d \mu(s)\right| \leqslant r\left\|\mu_{f}\right\|(X)=r\|f\|_{1}
$$

thus $\|Q f\|_{1}=\left\|\mu_{Q f}\right\|(X) \leqslant r\|f\|_{1}$.

THEOREM 3. Let $A=\int \lambda d \dot{E}(\lambda)$ be a bounded scalar operator defined on a Banach space $X$ with $\sigma(A)=T$. For $g \in X$, let $\mu(\cdot)=E(\cdot) g$. Define $U$ : $\mathcal{L}^{\mathrm{l}}(T, \mu) \rightarrow X$ by $U f=\int f d \mu$ and $Q: \mathcal{L}^{1}(T, \mu) \rightarrow \mathcal{L}^{1}(T, \mu)$ by $Q f(s)=s f(s)$. Then $U$ is continuous and the following diagram commutes:

$$
\begin{array}{ccc}
X & \stackrel{U}{\leftarrow} & \mathcal{L}^{1}(T, \mu) \\
A \downarrow & & \downarrow Q \\
X & \stackrel{U}{\leftarrow} & \mathcal{L}^{1}(T, \mu)
\end{array}
$$


Proof. For any polynomial $p \in \mathfrak{L}^{1}(T, \mu)$ we have $U p=p(A)$ so that

$$
A U p=A p(A)=U Q p .
$$

Since the polynomials are dense in $\mathcal{L}^{1}(T, \mu)$, the diagram commutes provided $U$ and $Q$ are continuous.

Since $A$ is bounded, $\operatorname{supp}(\mu)[\subset \sigma(A)]$ is bounded. Thus $Q$ is continuous by Theorem 2. That $U$ is continuous follows from

$$
|U f|=\left|\int f d \mu\right| \leqslant \sup _{E \subseteq T}\left|\int_{E} f d \mu\right|=\sup _{E \subseteq T}\left|\mu_{f}(E)\right| \leqslant\left\|\mu_{f}\right\|(T)=\|f\|_{1} .
$$

THEOREM 4. Let $A=\int \lambda d E(\lambda)$ be a scalar operator on a Banach space $X$ with $T=\sigma(A)$. If there exists a vector $g \in X$ so that $\mu(\cdot)=E(\cdot) g$ is full, then $A$ is similar to the multiplication operator on a factor space of $\mathfrak{L}^{\infty}(T, \mu)$.

PRoof. Since $\mathfrak{L}^{\infty}(T, \mu) \subset \mathfrak{L}^{1}(T, \mu)$ the diagram (2) above commutes with $\mathfrak{L}^{\infty}(T, \mu)$ replacing $\mathfrak{L}^{\prime}(T, \mu)$. By (1) we see that $U$ is continuous with the topology of $\mathscr{L}^{\infty}(T, \mu)$. Let $N$ be the null-space of $U$. Since $A U=U Q$, then $U f=0$ implies $U(Q f)=0$; thus $\hat{Q}: \mathfrak{L}^{\infty}(T, \mu) / N \rightarrow \mathfrak{L}^{\infty}(T, \mu) / N$ defined by $\hat{Q}[f]=[Q f]$ is well defined. Since $\mu$ is full and

$$
\{f \mid f \text { is measurable and } f(s) \in[0,1] \text { for all } s \in T\} \subseteq \mathscr{L}^{\infty}(T, \mu),
$$

the range of $U$ is all of $X$, and $\hat{U}: \mathscr{L}^{\infty}(T, \mu) / N \rightarrow X$, defined by $\hat{U}[f]=U f$, is bijective with a continuous inverse (ensured by the Open Mapping Theorem). By the commutativity of (2), it follows that $A=\hat{U} \hat{Q} \hat{U}^{-1}$.

\section{REFERENCES}

1. N. I. Akhiezer and I. M. Glazman, The theory of linear operators in Hilbert space, Ungar, New York, 1963.

2. N. Dunford and J. T. Schwartz, Linear operators, Interscience, New York, 1963.

3. I. Kluvanek and G. Knowles, Vector measures and control systems, New York, 1976.

Department of Mathematics, North Carolina State University, Raleigh, North Carolina 27607 (Current address of G. D. Faulkner)

Current address (J. E. Huneycutt, Jr.): Xonics, 1700 Old Meadow Road, McLean, Virginia 22101 